Oral presentation

Open Access

\title{
Functional evaluation of patients treated with osteochondral allograft transplantation for post-traumatic ankle arthrosis L Berti $^{1}$, MG Benedetti*1, F Vannini ${ }^{2}$, T Sforza ${ }^{1}$, L Conti ${ }^{1}$ and S Giannini ${ }^{1}$
}

\author{
Address: ${ }^{1}$ Movement Analysis Laboratory, Istituto Ortopedici Rizzoli, Bologna, Italy and ${ }^{2}$ Department of Orthopaedic Surgery, Istituto Ortopedici \\ Rizzoli, Bologna, Italy \\ Email: MG Benedetti* - benedetti@ior.it \\ * Corresponding author
}

from Ist Congress of the International Foot \& Ankle Biomechanics (i-FAB) community

Bologna, Italy. 4-6 September 2008

Published: 26 September 2008

Journal of Foot and Ankle Research 2008, I(Suppl I):O52 doi:I0.I I86/I757-I I46-I-SI-O52

This abstract is available from: http://www.jfootankleres.com/content/I/SI/O52

(c) 2008 Berti et al; licensee BioMed Central Ltd.

\section{Introduction}

Osteochondral allograft ankle transplantation [1] seems to provide a very innovative and viable alternative for the treatment of post-traumatic ankle arthritis in selected individuals, mostly in young and active patients. The rationale behind this surgical procedure is essentially the transplantation of intact organ as hyaline articular cartilage, into a diseased or damaged area of the joint resulting in improved function [2]. The aim of our study was to evaluate functional outcome of young and active patients that underwent osteochondral allograft ankle transplantation by means of gait analysis.

\section{Methods}

Eight patients affected by grade III post-traumatic arthritis were assessed pre-operatively and at the mean follow-up of 12 months. In all cases a fresh osteochondral bipolar resurfacing of the ankle was performed. Clinical evaluations were performed according to the American Orthopaedic Foot and Ankle Society (AOFAS) score. All patients were also studied with gait analysis using the VICON (Oxford, UK) stereophotogrammetric system to evaluate kinematics data and two force platform (Kistler Instrumente AG, Switzerland) to evaluate ground reaction forces. Using electromyography (Zero Wire, Aurion) we analysed the bilateral activity of four muscles of lower limbs (rectus femoris, biceps femoris, gastrocnemius, tibialis anterior). Results were compared with those of a control group, consisting of 10 healthy subjects.

\section{Results}

Clinical AOFAS score was significantly higher at twelve months after surgery (pre-surgery $43.8 \pm 14.0$; post-surgery $77.1(7.8, \mathrm{p}<0.0005)$. Gait analysis showed a wider range of motion in the sagittal plane (increased maximum dorsiflexion in stance phase, plantarflexion at toe off, maximum plantarflexion in swing phase) and improved maximum vertical force at loading response after surgery. The EMG pattern of muscle activity became more physiological in the post-operative analysis, even if most patients showed the persistence of co-contractions of gastrocnemius and tibialis anterior muscles during the first half of the stance phase.

\section{Conclusion}

Short-term functional evaluation of the osteochondral allograft ankle transplantation showed satisfactory results for these patients. The integrated gait analysis revealed only few abnormalities in kinematics, kinetics, and EMG. The persisting tibialis anterior activity during stance could be related to proprioception deficit and could be improved by time and exercise. It will be important to reevaluate these patients also at longer follow-ups in order to confirm these positive results.

\section{References \\ I. Kim CW, et al.: Foot Ankle Int 2002, 23:1091-I 102. \\ 2. Gross AE, et al.: Foot Ankle Int 200I, 22:385-39l.}

